\title{
Correction to: Modelling the HIV-Associated TB Epidemic and the Impact of Interventions Aimed at Epidemic Control
}

\author{
P. J. Dodd, Ronaldo de Carvalho Augusto, David Roquis, \\ Marion A. L. Picard, Cristian Chaparro, Celine Cosseau, \\ and Christoph Grunau
}

\section{Correction to: \\ Chapter 3 in: I. Sereti et al. (eds.), HIV and Tuberculosis, https://doi.org/10.1007/978-3-030-29108-2_3}

Unfortunately, the original version of this chapter was published as a non-open access chapter. This chapter has been changed to open access. Hence, the chapter "Modelling the HIV-Associated TB Epidemic and the Impact of Interventions Aimed at Epidemic Control" is now available open access under a Creative Commons Attribution 4.0 International License via link.springer.com.

In addition, the following acknowledgments section was not included in the original version of the chapter and has been included at the end of the text.

Acknowledgments This publication was produced by TREATS which is part of the EDCTP2 programme supported by the European Union (grant number RIA2016S-1632-TREATS). The views and opinions of the authors expressed herein do not necessarily state or reflect those of EDCTP.

\footnotetext{
The updated online version of this chapter can be found at https://doi.org/10.1007/978-3-030-29108-2_3
} 